\section{A control program for a Glace-Holmes speech synthesizer}

\author{
YOSHIYUKI HORII \\ Department of Audiology and Speech Sciences \\ Purdue University, West Lafayette, Indiana 47907
}

Synthetic speech provides unique opportunities to investigate important acoustic cues in speech signals and the nature of speech perception in general. The program, SYNG, described here is a utility program which controls a parallel-resonance terminal-analog speech synthesizer JAWORD (Glace, 1968). The synthesizer requires up to 11 inputs, 1 of which determines types of excitation (noise source, pulse source, or combination of the two), 1, fundamental frequency, and the remaining 9 , the formant frequencies and their amplitudes. Synthesizer control by computer essentially involves (1) preparation of sets of 11 parameter values, each set representing one time segment of speech, and (2) sending these sets of values to the synthesizer through digital-to-analog converters under programmed timing control.

Input/Output. Input data, from a card reader, Teletype, or magnetic tape unit, specify each set of 11 values corresponding to a 10 -msec sample of speech. An array of parameters for 600 time samples, which is equivalent to $6 \mathrm{sec}$ of speech, can be accommodated at one time. With the use of disk storage, 20 such parameter arrays can be stored and synthesized in any order, extending the system capacity to handle up to $120 \mathrm{sec}$ of speech. An option is also available to synthesize SOUGHS (Society of Users of Glace-Holmes Synthesizer) data punched on cards.

When parameter values of some time segments are not available, the program provides either linear or exponential interpolation to generate the missing information in a manner similar to that described by Rabiner (1968). The program then converts the successive sets of parameter values into equivalent voltages that are transferred to the synthesizer. The transfer rate can be specified at the time of execution, so that the synthesized speech can be compressed or stretched in time. A silent interval between the repetitions is also specifiable. On-going synthesis halts upon detection of an interrupt given manually by pressing a control button, at which time the user is given the several procedural options. For example, he can listen to any portion of the entire utterance at any desired transfer rate, he can obtain a printout of arrays of parameter values for any portion, or he can edit one or more parameter values for any segment. The edited portion can be synthesized alone or in the context of the entire utterance, or its parameter arrays can be written out and/or remodified. If desired, parameter values can be displayed as a function of time on an oscilloscope (one parameter) or on a graphic level recorder (up to eight parameters).

The program is written in an interactive form so that instructions to the user are typed out on the Teletype preceding each action required, and messages are given when illegal actions are taken. In contrast to synthesis by rule, in which input data typically specify a sequence of phonetic elements and accent and intonation codes (Mattingly, 1968), the present program utilizes parametric descriptions of utterances supplied by a primary recognition program (Hughes et al, 1969) which includes formant tracking and fundamental frequency tracking algorithms (Snow \& Hughes, 1969).

Program Language and Computer. The program is written in FORTRAN IV for a CDC 1700 computer with 16 channels of digital-to-analog conversion capability.

Availability. The listing of the program is available free on request from the author.

\section{REFERENCES}

Glace, D. A. Parallel resonance synthesizer for speech research. Journal of the Acoustical Society of America, 1968, 44, 391

Hughes, G. W., House, A. S., \& Li, K.-P. Research on word spotting. Final Report, Contract F 19628-67-C-0276, Purdue Research Foundation, 1969.

Mattingly, I. G. Synthesis by rule of general American English. Supplement to Status Report on Speech Perception, Haskins Laboratories, April 1968.

Rabiner, L. R. Speech synthesis by rule: An acoustic domain approach. Bell System Technical Journal, 1968, 47, 17-37.

Snow, T. B., \& Hughes, G. W. Fundamental frequency estimation by harmonic identification. Journal of the Acoustical Society of America, 1969, 45, 316.

Behavior Research Methods \& Instrumentation 1974, Vol. 6 (5), 505

\section{The change agent game (A computerized game in BASIC for use on time sharing terminals)}

\author{
CHARLES B. WEINBERG \\ Stanford University Graduate School of Business \\ Stanford, California 94305
}

The change agent game asks the student to adopt the role of a change agent whose goal is to secure adoption of an innovation by individuals in a rural village. The student has two broad strategies available: (1) to obtain information about the villagers' behavior, and (2) to select diffusion methods to motivate receivers to adopt the innovation. Information obtained from the first strategy, if properly used, is helpful in the selection of diffusion methods. The effectiveness of the strategies is based on results reported by Rogers (1971) and is represented by nonlinear response functions. The effectiveness is modified by random events which are modeled by a Monte Carlo simulation. The computerized game is based on a simpler pencil and paper game developed by Rogers.

The objectives of using the game are (1) to promote learning about the diffusion of innovation process, and (2) to show the value of a mangement approach to the change agent process. The game has been successfully used in a "social marketing" course taught by the author. Students are assigned to play the game on their own and results are discussed in a 1-h class session.

Computer and Language. Input and output are on a time sharing terminal. The game is programmed in BASIC and has been run successfully on an HP2000C.

Availability. The description of the game is divided into two parts: (1) The Change Agent Game, Players Manual, Stanford GSB, Technical Report No. 33 (12 pages), and (2) Instructor's Guide to the Change Agent Game, Stanford GSB, Technical Report No. 34 (13 pages). The Instructor's Guide contains the BASIC program listing. The reports are available at a cost of $\$ 1.50$ each from Technical Reports Editor, Graduate School of Business, Stanford University, Stanford, California 94305.

\section{REFERENCE}

Rogers, E. M., with Shoemaker, F. F. Communication of innovations. New York: Free Press, 1971. 